\title{
Early-onset progressive encephalopathy with migrant continuous myoclonus
}

INSERM

\section{Source}

INSERM. (1999). Orphanet: an online rare disease and orphan drug data base. Early-onset progressive encephalopathy with migrant continuous myoclonus. ORPHA:1943

A rare infantile epilepsy syndrome characterized by initially focal continuous myoclonus (lasting from dozens of minutes to hours), which later progresses to prolonged bilateral myoclonic seizures and generalized tonic-clonic seizures, and early-onset progressive encephalopathy, manifesting with hypotonia, ataxia and cortical atrophy. There have been no further descriptions in the literature since 1996. 